Utah State University

DigitalCommons@USU

$1-1-2009$

\title{
Characterizing the Performance of Liquid Oxygen in a Magnetic Fluid Management System
}

Jeffrey C. Boulware

Heng Ban

Scott Jensen

Steve Wassom

Follow this and additional works at: https://digitalcommons.usu.edu/sdl_pubs

\section{Recommended Citation}

Boulware, Jeffrey C.; Ban, Heng; Jensen, Scott; and Wassom, Steve, "Characterizing the Performance of Liquid Oxygen in a Magnetic Fluid Management System" (2009). Space Dynamics Lab Publications. Paper 21.

https://digitalcommons.usu.edu/sdl_pubs/21

This Article is brought to you for free and open access by the Space Dynamics Lab at DigitalCommons@USU. It has been accepted for inclusion in Space Dynamics Lab Publications by an authorized administrator of DigitalCommons@USU. For more information, please contact digitalcommons@usu.edu.

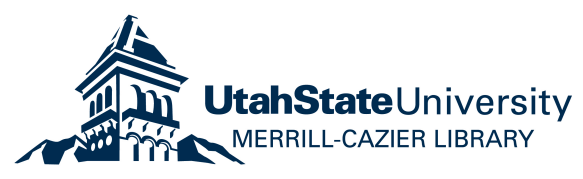




\title{
Characterizing the Performance of Liquid Oxygen in a Magnetic Fluid Management System
}

\author{
Jeffrey C. Boulware ${ }^{1}$ and Heng Ban ${ }^{2}$ \\ Utah State University, Logan, Utah, 84341 \\ Scott Jensen ${ }^{3}$, Steve Wassom ${ }^{4}$ \\ Space Dynamics Laboratory, Logan, Utah, 84341
}

\begin{abstract}
The strong paramagnetic susceptibility of liquid oxygen (LOX) has established it as a good candidate for a cryogenic magnetic fluid system. While these properties have been defined for several decades, the continuing advancement and requirements of space technology will soon find a suitable application for a magnetic fluid system which can operate reliably and efficiently. Testing has begun on the dynamics of LOX when applied to electrically-induced steady and varying magnetic fields within a solenoid. The performance of LOX as a working fluid was characterized by its operability and sustainable pressure before breakdown. This paper presents numerical and experimental data on the performance characteristics of LOX in a magnetic fluid management system.
\end{abstract}

\section{Nomenclature}

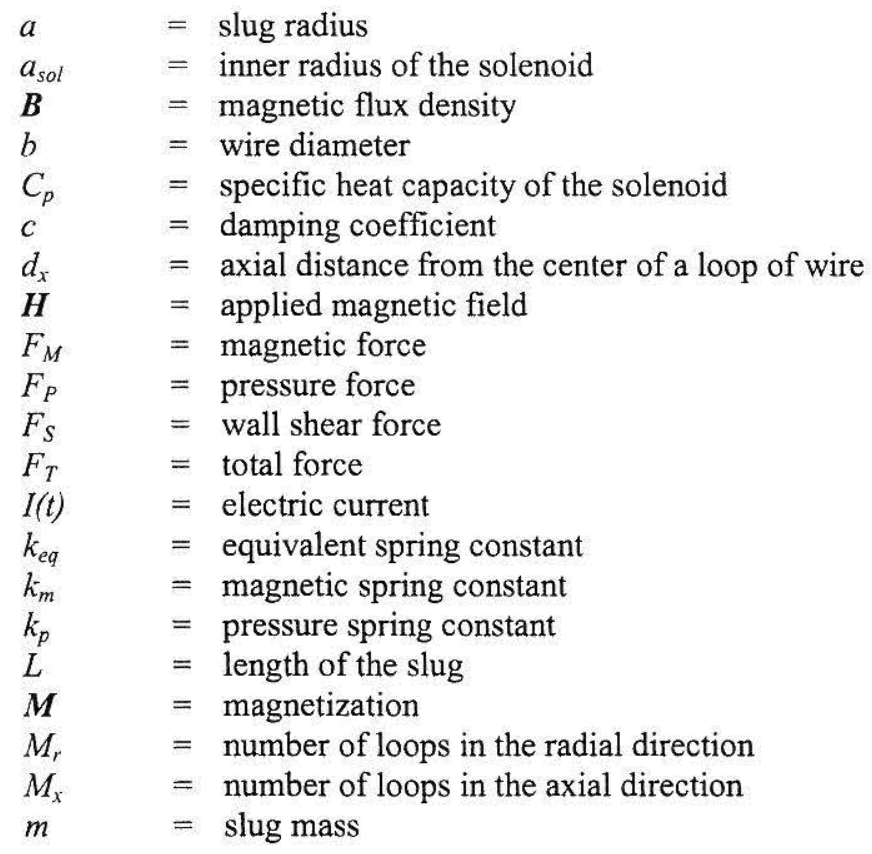

\footnotetext{
${ }^{1} \mathrm{PhD}$ Candidate, Mechanical \& Aerospace Engineering, 4130 Old Main Hill, Logan, Utah 84321, 435-797-4014, Student Member.

${ }^{2}$ Associate Professor, Mechanical \& Aerospace Engineering, 4130 Old Main Hill, Logan, Utah 84321, 435-7972098, Member.

${ }^{3}$ Co-Director, Thermal Management Technologies, Space Dynamics Laboratory, 1695 N. Research Park Way, North Logan, Utah 84341, 435-797-4516, Member.

${ }^{4}$ Group Leader, Mechanical/Thermal Engineering, Space Dynamics Laboratory, 1695 N. Research Park Way, North Logan, Utah 84341, 435-797-4672
} 


$\begin{array}{ll}m_{\text {sol }} & =\text { mass of the solenoid } \\ P(t) & =\text { power } \\ P_{i} & =\text { initial system pressure } \\ R(t) & =\text { electrical resistance } \\ R e & =\text { Reynolds number } \\ r_{\text {loop }} & =\text { radius of a loop of wire } \\ T(t) & =\text { temperature of the solenoid } \\ t & =\text { time } \\ x & =\text { slug displacement } \\ \dot{x} & =\text { slug velocity } \\ \ddot{x} & =\text { slug acceleration } \\ x_{e q . s s} & =\text { steady-state equilibrium point } \\ x_{m} & =\text { magnetism-only equilibrium point } \\ x_{p} & =\text { pressure-only equilibrium point } \\ V & =\text { applied voltage } \\ \alpha & =\text { coefficient of thermal resistance } \\ \chi & =\text { volumetric susceptibility } \\ \Delta x & =\text { wire spacing in the axial direction } \\ \Delta r & =\text { wire spacing in the radial direction } \\ \Delta p & =\text { differential pressure about the slug } \\ \eta & =\text { dynamic viscosity } \\ \mu_{o} & =\text { permeability of free space } \\ \rho & =\text { density of liquid oxygen } \\ \tau_{w} & \text { wall shear stress }\end{array}$

\section{Introduction}

$\mathrm{T}$ HE practical application of magnetic fluids has been proven and has found many industrial uses in areas such as audio speakers, digital data storage, and resonance imaging. Even as a working fluid, many theoretical ideas have become textbook material as shown by Rosensweig ${ }^{1}$ and Odenbach ${ }^{2}$. In the medical and computer industries, the works of $\mathrm{Seo}^{3}, \mathrm{Krauss}^{4}, \mathrm{Liao}^{5}$, and $\mathrm{Hatch}^{6}$ have turned concept into reality. Each of these investigators has successfully created an actuator for propagating an artificial ferrofluid by means of magnetic fields only. And while these artificial ferrofluids have tailorable qualities, their applicability in space is severely limited due to harsh environmental constraints. Liquid oxygen (LOX), on the other hand, is already commonly used in space systems for life support, thermal management, and propulsion. At its boiling point of $90 \mathrm{~K}$, LOX has the highest known paramagnetic susceptibility of pure fluids at 0.0035 . Although even an inexpensive ferrofluid can be 30 times stronger, the presence of nanoparticles and limited temperature range prevents their widespread applicability in space.

The current study was intended to determine the viability of LOX in a magnetic fluid system through experimental and theoretical analyses. A previous test by Youngquist ${ }^{7}$ proved the viability of displacing a LOX column against gravity with an electrically-driven solenoid, whereas the current effort focused on displacing a slug in a closed system and measuring the resultant pressure change from the reduced volume. With helium as the surrounding gas, an isothermal ideal gas compression assumption could be used. Figure 1 shows that as the slug moved to the right (considered hereafter as "upstream") in the closed system, the downstream volume, $V_{D S}$, decreased and upstream volume, $V_{U S}$, increased causing a reciprocal reaction for the downstream and upstream pressures, $P_{D S}$ and $P_{U S}$. The pressure fluctuations could be used to measure displacement and the performance of LOX was characterized by its behavior with specific test parameters. 


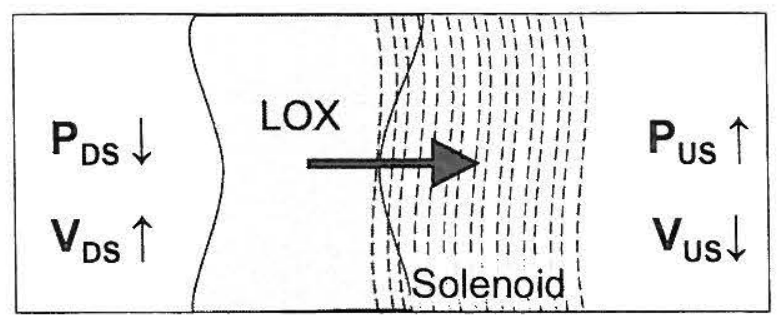

Figure 1. LOX slug displacement in a closed system.

\section{Experimental Setup}

An apparatus was designed to displace a slug of LOX via electrically-pulsed solenoids as shown in Figure 2. The test section and heat exchanger were completely submerged in liquid nitrogen to mitigate thermal acoustic oscillations. Helium acted as the surrounding gas for the LOX slug due to its inertness with oxygen and low boiling point. The total system volume was $340 \mathrm{~cm}^{3}$, although only about $1.8 \mathrm{~cm}^{3}$ existed downstream of the test section. This allowed for high-pressure changes when the slug was displaced. Pressure sensors upstream and downstream of the LOX slug in the test section recorded data at $5 \mathrm{kHz}$ with a $175 \mathrm{~Pa}(0.025 \mathrm{psi})$ resolution, thus relating to a displacement precision of $0.8 \mathrm{~mm}(0.02 \mathrm{in})$. The solenoids and test section were created by wrapping copper wire on plastic bobbins and then sliding them over a $1.9 \mathrm{~mm}$ ID quartz tube.

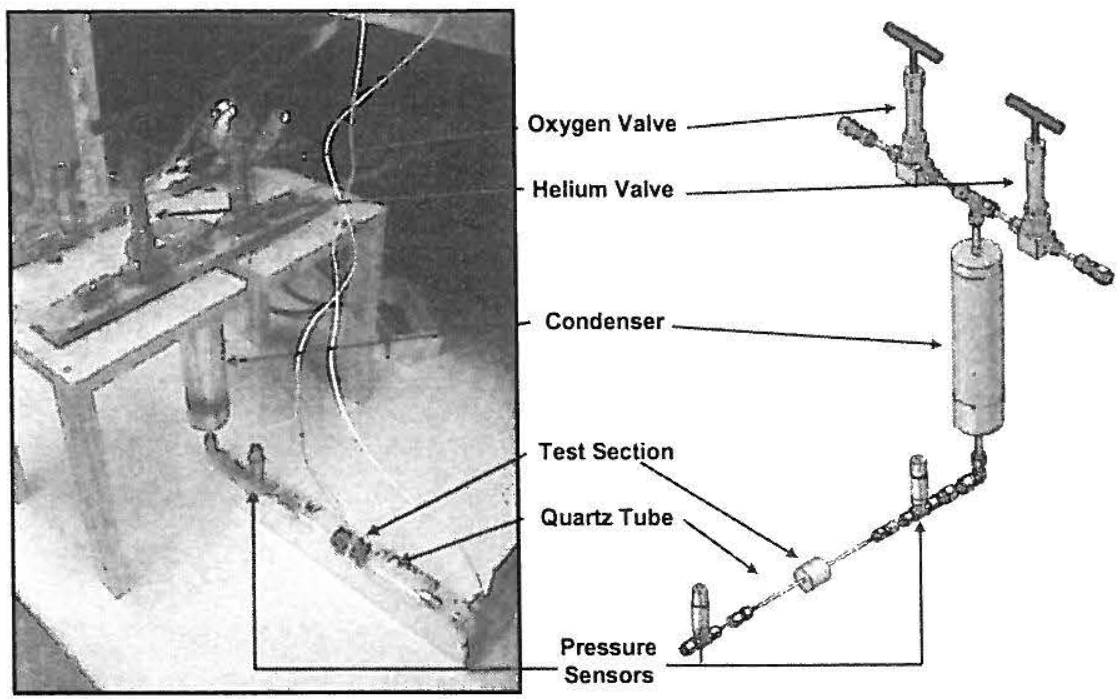

Figure 2. Photograph and CAD drawing of experimental apparatus.

The quartz tube interfaced with stainless steel plumbing through removable Teflon heat shrink tubing that allowed for easy interchangeability of the test section. The maximum absolute pressure in the system did not exceed $172 \mathrm{kPa}(25 \mathrm{psi})$ and all fittings provided a helium-tight seal with leak rates less than $20 \mathrm{~Pa} / \mathrm{min}(0.003 \mathrm{psi} / \mathrm{min})$. When submerged in liquid nitrogen, the solenoids produced up to $1.1 \mathrm{~T}$ with a $900 \mathrm{~W}$ power supply. The operating pressure was maintained above atmospheric pressure to prevent a cold vacuum, but held within a gauge pressure of $325 \mathrm{kPa}(5 \mathrm{psi})$ to maintain safe operating levels. Prior to each test, the length of the slug and its initial position relative the solenoid were recorded. The LOX slug formed a concave meniscus and the edges were measureable within $1.6 \mathrm{~mm}(0.0625 \mathrm{in})$ resolution.

\section{Theory}

A fundamental understanding of the dynamics of a LOX slug must be achieved in order to establish its predictability in a magnetic fluid system. By analyzing the system through a force balance, the dominant forces can 
be used to describe the dynamics mathematically and simulated in a numerical algorithm. Once the solenoid was powered, the slug oscillated about an equilibrium point and the system could be compared to a perturbed pneumatic system with exponential damping. Derivations by Rosensweig ${ }^{1}$ were used to develop the force balance, and derivations by $\operatorname{Inman}^{8}$ were used to develop the perturbation analysis.

\section{A. Force Balance}

The dominant forces on the LOX slug were due to magnetism, pressure differences, and wall shear. Gravitational and surface tension forces were considered to be negligible for the test apparatus, but may need to be considered for a different geometry. A free body diagram of the dominant forces is shown in Figure 3.

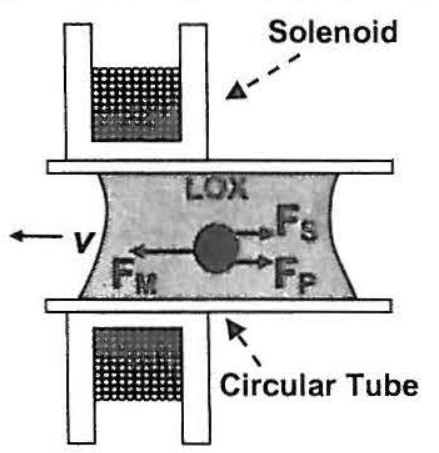

Figure 3. Free body diagram of LOX slug dynamics.

Note that in Figure 3 the slug is not at rest. The magnetic force, $F_{M}$, is greater than the combined shear force, $F_{S}$, and pressure force, $\mathrm{F}_{\mathrm{P}}$, and results in a net velocity to the left. As the slug's momentum moves its center past the solenoid, the magnetic force changes direction and the slug is pushed in the other direction. The shear force dampens the slug's velocity and an eventual steady state will balance only the magnetic and pressure forces as the slug reaches an equilibrium point. If the pressure differential about the slug is negligible, it will center itself on the solenoid since magnetism acts as a body force. Mathematically, the force balance is expressed in Eq. (1) as

$$
m \ddot{x}=F_{P}(x)+F_{M}(x)+F_{S}(\dot{x})
$$

Thus, the acceleration of the slug is due to the net force upon it. The forces must be individually analyzed to determine their net effect. The pressure force results from the differential pressure on either side of the slug as

$$
F_{P}=\pi a^{2} \Delta p
$$

The change in pressure results from the closed volumes on either side of the slug being compressed and expanded. The magnetic force can be found by the Kelvin force density through the divergence of the Maxwell stress tensor as

$$
f_{m}=\mu_{0}(\boldsymbol{M} \cdot \nabla) \boldsymbol{H}
$$

For soft magnetic materials, volumetric susceptibility is the ratio of the magnetization vector to the applied field vector, $\chi=\boldsymbol{M} / \boldsymbol{H}$. By substituting for $\boldsymbol{M}$, using the vector identity, $\boldsymbol{H} \cdot \nabla \boldsymbol{H}=\frac{1}{2} \nabla(\boldsymbol{H} \cdot \boldsymbol{H})-\boldsymbol{H} \times(\nabla \times \boldsymbol{H})$, and noting that Ampere's Law cancels out the curl of the applied field, Eq. (3) can be reduced to

$$
f_{m}=\frac{1}{2} \mu_{0} \chi \nabla \boldsymbol{H}^{2}
$$

With a constant temperature, the relative permeability, $\mu$, also remains constant. The relative permeability is the ratio between the magnetic flux density, $\boldsymbol{B}$, and applied magnetic fields, $\mu=\boldsymbol{B} / \boldsymbol{H}$, but can also expressed in terms of volumetric susceptibility, $\mu=\mu_{0}(I+\chi)$. Given these relations, the Kelvin force density in the axial direction is 


$$
f_{m, x}=\frac{1}{2 \mu_{0}} \frac{\chi}{(1+\chi)^{2}} \frac{d}{d x} B_{x}^{2}
$$

Where the subscript $x$ denotes the axial direction. The differential term considers the ends of the slug and when integrated over the entire volume, the force due to magnetism in the axial direction, $\mathrm{F}_{\mathrm{M}}$, is

$$
F_{M}=\frac{\pi a^{2}}{2 \mu_{0}} \frac{\chi}{(1+\chi)^{2}}\left(B_{x, U S}{ }^{2}-B_{x, D S}{ }^{2}\right)
$$

Where the subscripts $U S$ and $D S$ denote the upstream and downstream directions. The magnetic flux density on the axis of a solenoid can be predicted by summing all the wire loops in the solenoid. The individual loop contribution is

$$
B_{x, \text { loop }}=\frac{1}{2} I(t) \mu_{0} r_{\text {loop }}^{2}\left(r_{\text {loop }}^{2}+d x^{2}\right)^{-3 / 2}
$$

The magnetic flux density resulting from the entire solenoid can be found with known number of loops and wire spacing. Resistance heating in the solenoid causes the magnetic field to decrease over time. By knowing the coefficient of thermal resistance for copper and measuring the overall specific heat of the solenoid, the current as a function of time could be calculated for a constant voltage through a series of equations as

$$
\begin{gathered}
P(t)=I^{2}(t) R(t) \\
T(t)=T_{0}+\frac{P(t)}{m_{s o l} c_{p}} t \\
R(t)=R_{0}\left(1+\alpha\left(T(t)-T_{0}\right)\right) \\
I(t)=V / R(t)
\end{gathered}
$$

Where the subscript 0 denotes the initial value. Time-stepping through Eqs. (8-11) outputs the temperature and current of the solenoid. Then, using Eq. (7), the magnetic force can be calculated over time for an individual loop.

Damping results from the force due to fluid shear on the wall, $\mathrm{F}_{\mathrm{S}}$, which is dependent on the wall shear stress of the transient LOX flow. As an approximation, a fully-developed pipe flow shear stress was used. White ${ }^{9}$ presents the classic relation for laminar wall shear stress and the Blasius correlation for turbulent wall shear stress, and an interpolative relation for the transient region in between could be developed. The force and wall shear stress are written as

$$
F_{S}=2 \pi a L \tau_{W}
$$

$$
\begin{array}{ll}
\tau_{w}=4 \dot{x} \eta / a & \mathrm{Re}<2000 \\
\tau_{w}=\frac{\rho \dot{x}^{2}}{2}(0.0153 \log (\mathrm{Re})-0.0424) & 2000 \leq \mathrm{Re} \leq 3000 \\
\tau_{w}=0.0396 \rho^{3 / 4} \dot{x}^{7 / 4} \eta^{1 / 4}(2 a)^{-1 / 4} & \mathrm{Re}>3000
\end{array}
$$

When Eqs. (2), (6), and (12) are substituted into Eq. (1), the equation of motion for the LOX slug becomes 


$$
m \ddot{x}=\pi a^{2} \Delta p+\frac{\pi a^{2}}{2 \mu_{0}} \frac{\chi}{(1+\chi)^{2}}\left(B_{x, U S}{ }^{2}-B_{x, D S}{ }^{2}\right)+2 \pi a L \tau_{w}
$$

\section{B. Perturbation Analysis}

Once the slug is disturbed from its equilibrium position, it experiences dampened oscillations and eventually returns to its steady-state equilibrium point. The oscillating mass is similar to a pneumatic piston and can be described by a perturbation analysis with position and velocity based forces. The basic unforced spring-massdamper equation and its solution are defined by

$$
\begin{gathered}
m \ddot{x}+c \dot{x}+k_{e q} x=0 \\
x(t)=X \exp \left(-\zeta w_{n} t\right) \cos \left(\omega_{d} t+\delta\right) \\
\omega_{n}=\sqrt{\frac{k_{e q} / m}{\zeta} \quad \zeta=c / 2 \sqrt{k_{e q} m} \quad \omega_{d}=\omega_{n} \sqrt{1-\xi^{2}}} \\
X=\sqrt{\frac{\left(v_{0}+\omega_{n} \zeta x_{0}\right)^{2}+\left(x_{0} \omega_{d}\right)^{2}}{\omega_{d}^{2}}} \quad \delta=\tan ^{-1}\left[\frac{x_{0} \omega_{d}}{v_{0}+\omega_{n} \zeta x_{0}}\right]
\end{gathered}
$$

The initial values for displacement and velocity, $\mathrm{x}_{0}$ and $\mathrm{v}_{0}$, are distinct to individual test runs. The upper and lower bounds of the oscillations are represented by Eq. (16) without the cosine term. The position-dependent pressure and magnetism forces act as parallel nonlinear springs and attribute to the spring constant as $\mathrm{k}_{\mathrm{eq}}=\mathrm{k}_{\mathrm{M}}+\mathrm{k}_{\mathrm{p}}$. The damping constant for the laminar region can be pulled from Eq. (13a) as

$$
c=8 \pi L \eta
$$

As the flow moves into transition and turbulent flow, the damping constant also becomes nonlinear, but the experimental data taken did not approach this limit. Although the bulk motion of the slug remains within the laminar regime, the theory does not account for internal flow dynamics.

\section{Nonmagnetized}

Without the presence of a magnetic field, the system is nearly linear. The upstream and downstream gas compression and expansion enforce a position-dependency on the spring constant; however, fluctuations on the order of 5-10 mm cause the spring constant to deviate from its mean by less than $1 \%$. The pressure-based spring constant is written as functions of the upstream and downstream pressure and volume changes as

$$
\begin{aligned}
k_{p} & =F_{p} / x \\
& =\frac{\pi a^{2}}{x}\left(P_{D S}-P_{U S}\right) \\
& =\frac{\pi a^{2}}{x} P_{i}\left(\frac{V_{D S, i}}{V_{D S}}-\frac{V_{U S, i}}{V_{U S}}\right) \\
& =\frac{\pi a^{2}}{x} P_{i}\left(\frac{V_{D S, i}}{V_{D S, i}+\pi a^{2} x}-\frac{V_{U S, i}}{V_{U S, i}-\pi a^{2} x}\right)
\end{aligned}
$$


As shown, the spring constant is inversely quadratic with position, but can be easily calculated in a numerical simulation. The equilibrium point for the nonmagnetized setup, $x_{p}$, was considered as the origin for the system dynamics.

\section{Magnetized}

When a magnetic force is present, the equilibrium position of the slug shifts. Furthermore, since the magnetic force is dependent on the position of the ends of the slug, the equilibrium position fluctuates with the mass oscillations. Figure 4 illustrates the forces at hand.

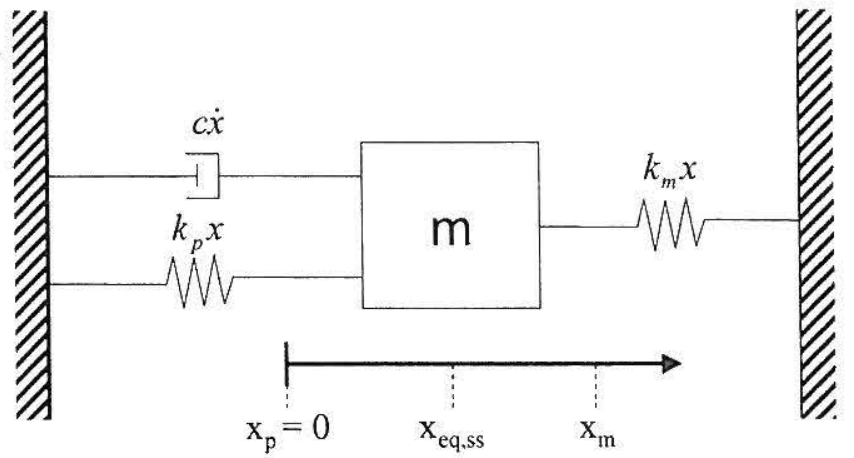

Figure 4. Spring-mass-damper system of an oscillating slug.

The positions marked $x_{p}$ and $x_{m}$ represent the equilibrium positions if only one of the spring forces were present and when combined, the steady-state equilibrium position exists somewhere between $x_{p}$ and $x_{m}$. For a linear system, the mass would oscillate about an equilibrium position, but because the magnetic force is nonlinear, the equilibrium position itself experiences oscillations. The damping force eventually stabilizes the oscillations and forces a steadystate equilibrium point. In a manner, the magnetic force acts as an external force, but since it is position-dependent, it is treated as a nonlinear spring.

Aside from the nonlinear spring constant, the magnetization also affects the damping force. Magnetoviscous effects have been heavily studied on ferrofluids, but the necessary data does not exist for LOX. Hilton ${ }^{10}$ presents the viscosity of nonmagnetized LOX as $284.9 \mu \mathrm{Pa} \cdot \mathrm{sec}$ at $77 \mathrm{~K}$ and $155 \mathrm{kPa}$, but for the current study, an empirical approximation was required.

Relative to $\mathrm{x}_{\mathrm{p}}$, the harmonic equation for the magnetized slug can be written as

$$
x(t)=X \exp \left(-\frac{4 \eta}{\rho a^{2}} t\right) \cos \left(\sqrt{\frac{k_{p}+k_{m}}{\pi a^{2} \rho L}-\left(\frac{4 \eta}{\rho a^{2}}\right)^{2}} t-\delta\right)+x_{e q, s s}\left(F_{P}(t), F_{M}(t)\right)
$$

The solution for the steady-state equilibrium point over time is a function of the temporal variations of the pressure and magnetic forces.

\section{Hydrostatic breakdown}

The force balance and perturbation analysis do not account for hydrostatic breakdown of the slug. As aforementioned, the one-dimensional analyses cannot account for surface tension or instabilities of the finite-length slug. Experiments by Perry ${ }^{11}$ confirm the theoretical predictions of ferrohydrodynamic breakdown as a balance of the magnetic and pressure forces. Beginning with the magnetic Bernoulli equation, the balance can be derived as

$$
\Delta p_{\max }=\frac{1}{2 \mu_{0}} \frac{\chi}{(1+\chi)^{2}}\left(B_{x, U S}^{2}-B_{x, D S}^{2}\right)
$$

Because the experimental data matched well with theory, it is determined that surface instability did not play a major role in the hydrostatic breakdown of his experiments. Thus, Eq. (22) can be used to find the limits of

American Institute of Aeronautics and Astronautics

$39^{\text {th }}$ AIAA Fluid Dynamics Conference

Flow control: Active, passive, and closed-loop flow control; actuators 
capability. For an actual LOX-based magnetic fluid system, the hydrostatic breakdown strength would be the most influential design parameter for system capability.

\section{Numerical Model}

The governing equations were discretized using a first-order finite-difference method for space and time. Given initial conditions of the position, velocity, and total force, a kinematic equation was used to find the slug's position at each time step. The discretized algorithms for calculating the pressure and magnetic forces are summarized in Eqs. (23-26).

$$
\begin{gathered}
F_{P, i}=\pi a^{2}\left(P_{D S, i}-P_{U S, i}\right) \\
F_{S, i}=2 \pi a L \tau_{w, i} \\
B_{x, U S, i}=\frac{\mu_{0} I_{i}}{2} \sum_{m=-\frac{1}{2}\left(M_{x}-1\right)}^{\frac{1}{2}\left(M_{x}-1\right)} \sum_{n=0}^{M_{r}-1} \frac{\left(a_{s o l}+n b \Delta r\right)^{2}}{\left[\left(a_{s o l}+n b \Delta r\right)^{2}+\left(x_{i}-m b \Delta x\right)^{2}\right]^{3 / 2}} \\
B_{x, D S, i}=\frac{\mu_{0} I_{i}}{2} \sum_{m=-\frac{1}{2}\left(M_{x}-1\right)}^{\frac{1}{2}\left(M_{\tau}-1\right)} \sum_{n=0}^{M_{r}-1} \frac{\left(a_{s o l}+n b \Delta r\right)^{2}}{\left[\left(a_{s o l}+n b \Delta r\right)^{2}+\left(x_{i}-L-m b \Delta x\right)^{2}\right]^{3 / 2}} \\
F_{M, i}=\frac{\pi a^{2}}{2 \mu_{0}} \frac{\chi}{(1+\chi)^{2}}\left(B_{x, U S, i}{ }^{2}-B_{x, D S, i}{ }^{2}\right)
\end{gathered}
$$

Where the subscript $i$ denotes the time step. The convention used was that positive displacement is in the upstream direction. With all three external forces determined, a total force on the slug could be formed.

$$
F_{T, i}=F_{P, i}+F_{M, i}-\operatorname{sgn}\left(\dot{x}_{i-1}\right) F_{S, i}
$$

where the sign function in the third term on the right hand side forced the shear component to always act in the opposite direction of velocity. Then, the left hand side of Eq. (14) was discretized and balanced with the average total force between time steps. After rearranging, the equation of motion was

$$
\dot{x}_{i}=\frac{F_{T, i}+F_{T, i-1}}{2 \rho \pi a^{2} L} \Delta t+\dot{x}_{i-1}
$$

The numerical simulation then cycled to the next time step and repeated the algorithm to calculate the pulse dynamics relative to a solenoid. Once the total relative dynamics were solved for a solenoid, they were converted to absolute coordinates for the entire test setup. The process was repeated throughout the entire pulsing sequence as shown in Figure 5. 


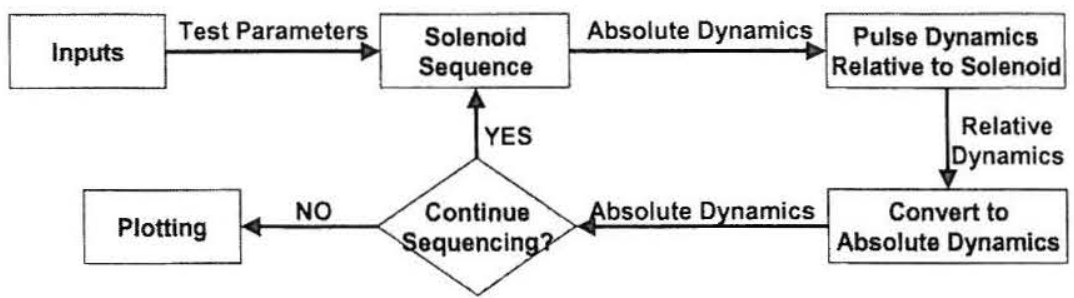

Figure 5. Numerical simulation block diagram.

\section{Results and Discussion}

\section{A. Single Pulse Dynamics}

The experiment produced interesting results for the slug dynamics. When power to the solenoids was switched on, the slug moved to a new position and oscillated about the new equilibrium point with decaying oscillation amplitude. The equilibrium point decreased with time as predicted by the resistance heating calculations, but did not oscillate itself. It is likely that the equilibrium oscillations were negligible compared to the overall oscillations and were not observable through the data. Figure 6 shows the downstream pressure over time and the results of the numerical simulation.

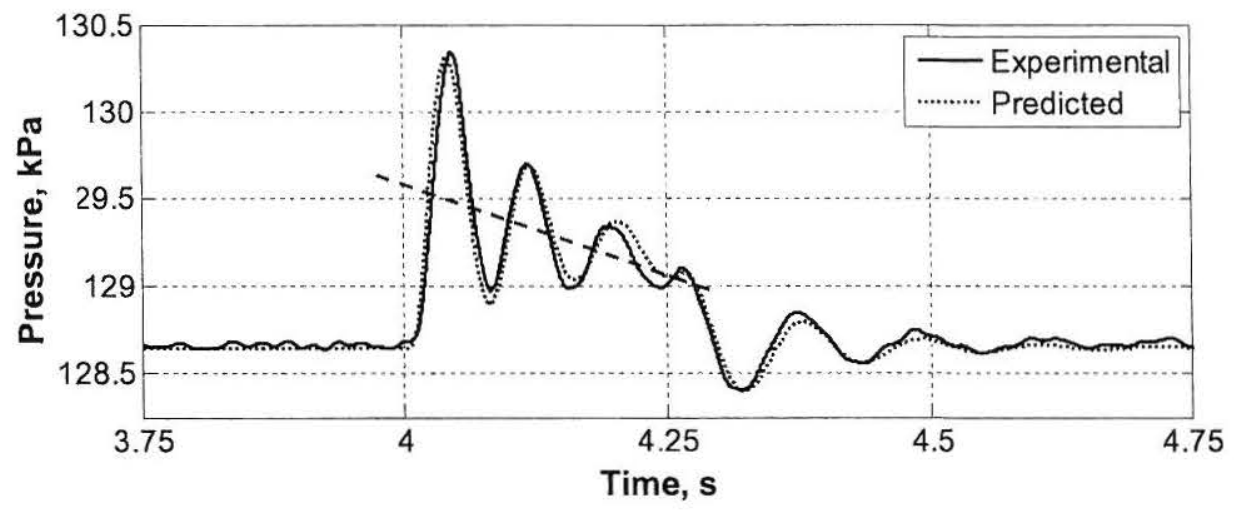

Figure 6. Downstream pressure response of a LOX slug propagated by an electrically-pulsed solenoid.

When power to the solenoid was switched off, the slug moved back to its neutral position and pressures on both sides of the slug equalized, again with the damped oscillations. Positive pressure indicates the slug was displaced towards the downstream end causing compression and the dashed line represents the decreasing mean displacement when the solenoid is powered. Figure 6 shows the downstream pressure response and numerical simulation of a 0.25 second, $1.1 \mathrm{~T}$ pulse on a $1.9 \mathrm{~cm}$ slug, using an effective slug length of $5.3 \mathrm{~cm}$. The effective slug length is calculated by the perturbation analysis to account for the unseen portions of the slug. Because the metal tubing in upstream and downstream sections of the slug prohibit visual confirmation of the amount of LOX, additional slugs may have formed. If so, the dynamics of the system must include these slugs and an effective slug length larger than the actual slug length must be used for the numerical model. The volume between the two slugs was assumed to be rigid.

The run in Figure 6 also indicated a viscosity 4.75 times that of nonmagnetized LOX at $77 \mathrm{~K}$. While the perturbation analysis accurately determined the effective viscosity, the unknown magnetoviscous effects and internal flow dynamics may have increased the damping in a manner other than wall shear, but the amount is unknown. The fully-developed shear stress does not take into account the oscillations of the slug. Schlichting ${ }^{12}$ presents the solution for an oscillating pressure profile in pipe flow, but actually results in lower shear since the specific parameters do not achieve a velocity overshoot. Instead, the problem must be analyzed as an oscillating wall on a finite length slug. Until a CFD model can be created which combines the magnetorheological effects, an effective viscosity must be used. The perturbation analysis allowed a good approximation for the effective viscosity and satisfied the damping requirements. In the future, cryogenic rheometers and computational fluid dynamics should be used to measure the 
unknown magnetoviscous effects and slug internal flow. The susceptibility dependence on temperature was not considered a source of uncertainty since its maximum relative change was less than $1 \%$.

The simulation was also dependent on the initial position of the slug. Figure 7 shows the relationship between the maximum pressure differential generated and initial position of the center of the slug relative to the solenoid. The solid line represents the simulation with an effective length of $10.5 \mathrm{~cm}$ and a viscosity 2.1 times the nonmagnetized value. The experimental data was for an actual slug length of $1.9 \mathrm{~cm}$ and the points marked with an " $\mathrm{x}$ " indicate runs that experienced a hydrostatic breakdown. The theory predicted that the breakdown should have occurred around $1.9 \mathrm{kPa}(0.27 \mathrm{psi})$ for the current setup, which is slightly higher than the data obtained. The difference is expectedly due to greater wire spacing than expected and resistance heating.

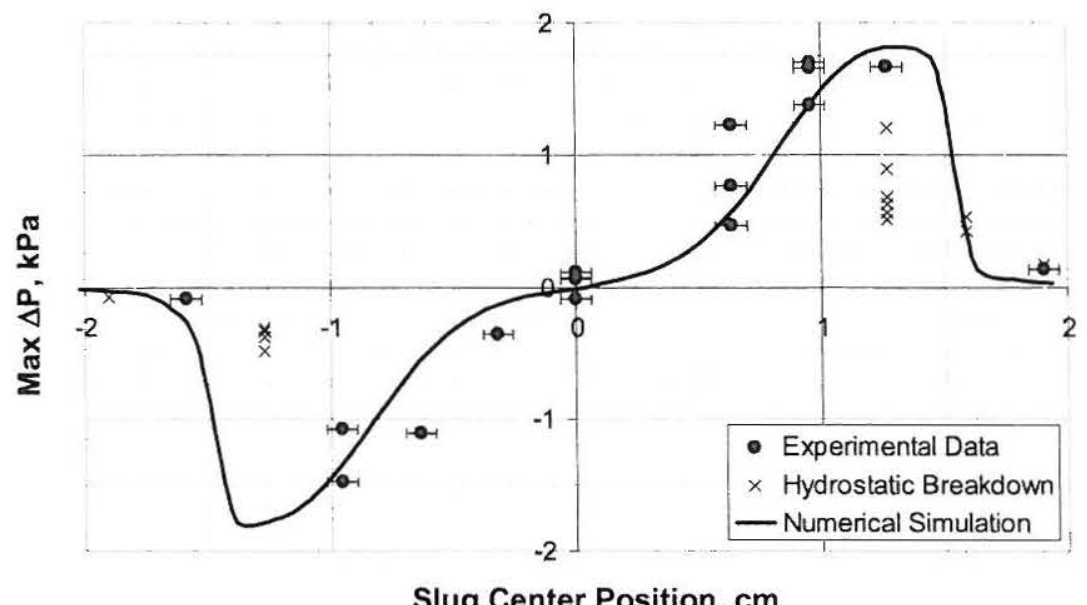

Figure 7. Experimental versus numerical pressure generated for a $1.9 \mathrm{~cm}$ slug.

The model was used to predict peak pressure trends with respect to slug length and position. Figure 8 shows the simulation's predictions on the maximum generated pressure differential as the slug length and center position vary. The simulation showed that the maximum pressure differential occurred when the slug was about twice the length of the solenoid and began with its closest edge just outside the solenoid. In a system free of shear, the pressure differential would continue to increase with the slug length and position.
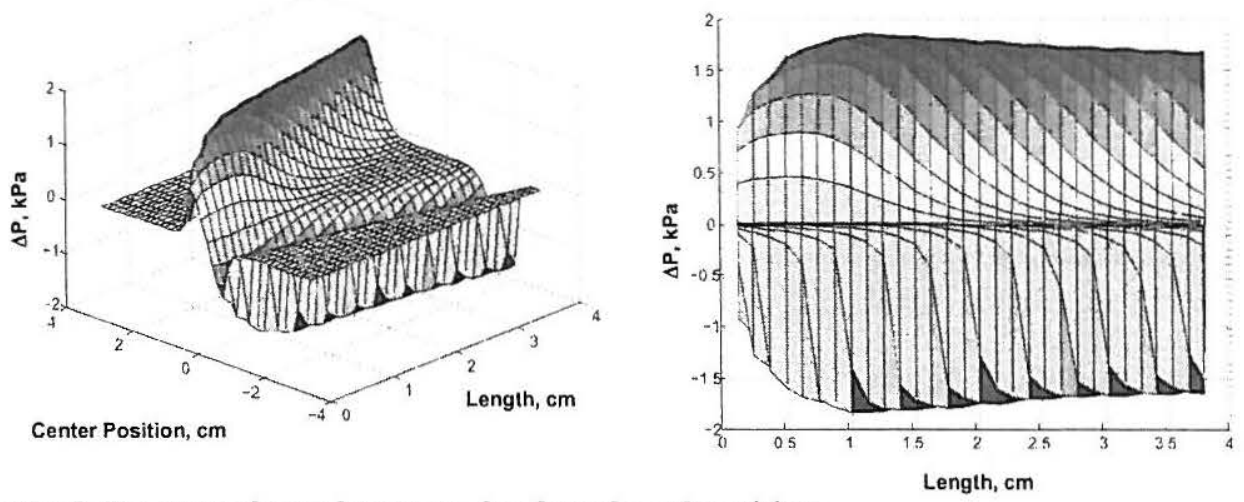

Figure 8. Pressure dependency on slug length and position.

\section{B. Multiple Pulse Dynamics}

As a higher order test, a $2.3 \mathrm{~cm}$ slug was pulsed between two solenoids at 0.25 seconds each from upstream to downstream. Figure 9 shows the correlation for the experimental data when using an effective slug length of 11.1 $\mathrm{cm}$ and a viscosity 2.1 times the nonmagnetized value. This result proved that the LOX slug could be moved from solenoid to solenoid indicating that multiple solenoids could be used to move and control the motion of a LOX slug for certain applications. 


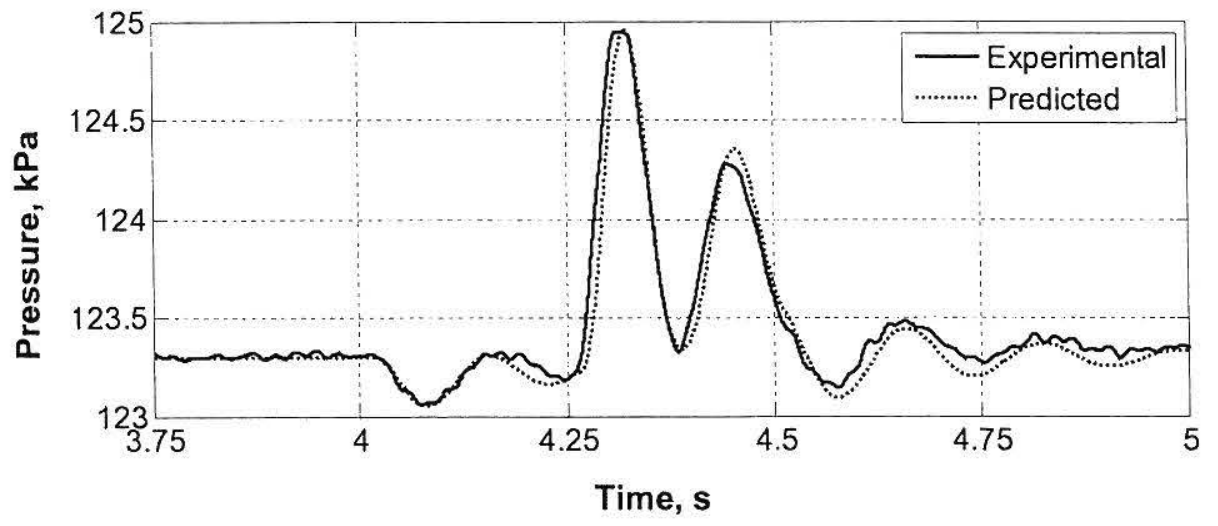

Figure 9. Downstream pressure correlation for multiple solenoids.

As seen, the simulation still predicts the slug dynamics well. The uncertainty increases with the number of pulses because the space between each solenoid adds another variable to the analysis. Disregarding the simulation, Figure 10 illustrates another multiple pulse test of a slug with an effective length $0 \mathrm{c} 9.5 \mathrm{~cm}$.
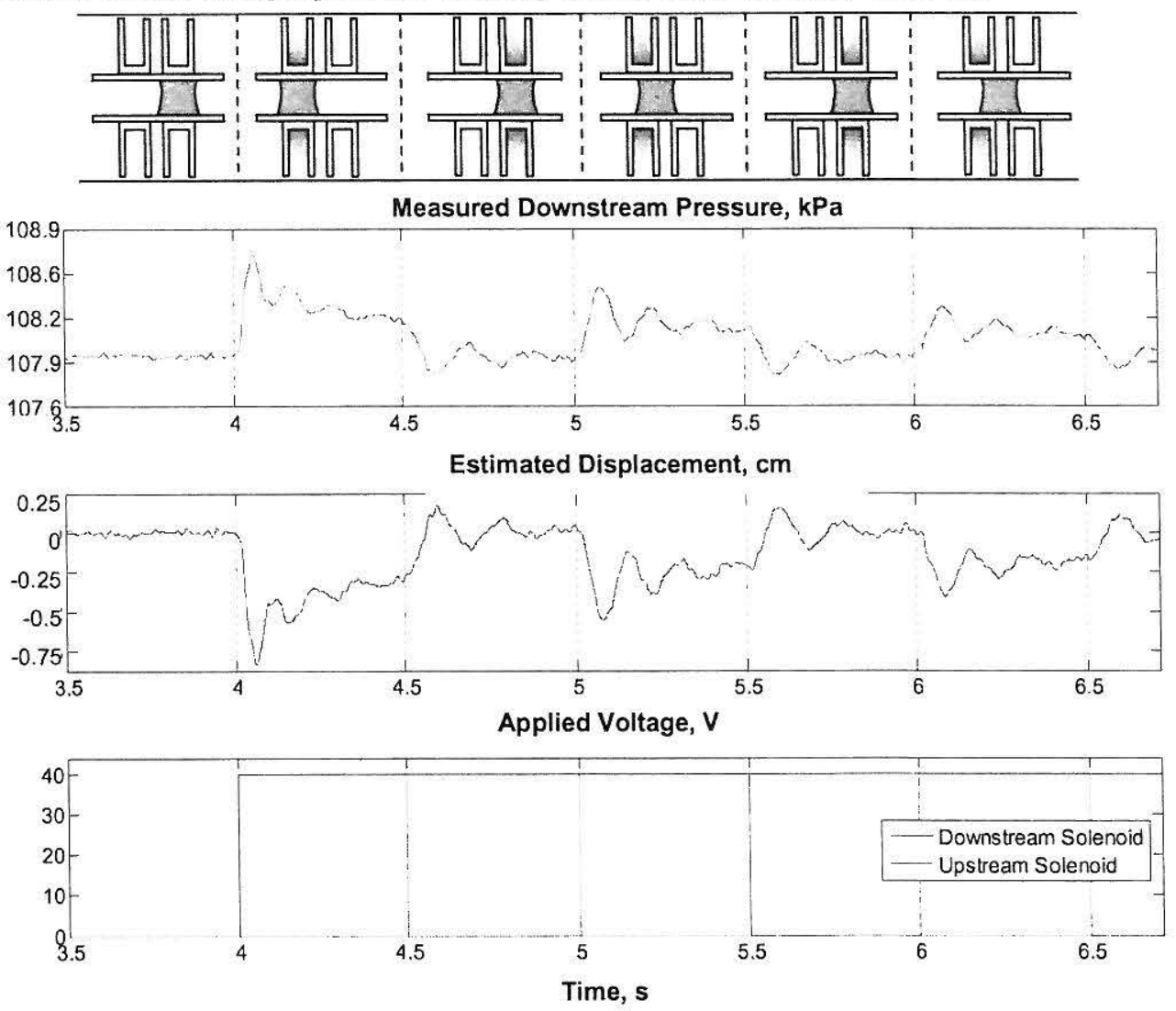

Figure 10. Multiple solenoid pulsing dynamics for a slug with an effective length of $9.5 \mathrm{~cm}$.

As the slug was transferred between the two solenoids, the downstream volume was compressed and decompressed giving an accurate measure of the displacement. As mentioned earlier, a pressure resolution of $175 \mathrm{~Pa}$ corresponds to a displacement resolution of about $0.8 \mathrm{~mm}$.

\section{Hydrostatic Breakdown}

Figure 7 showed the points at which a hydrostatic breakdown occurred. The breakdown was confirmed by the data as shown in Figure 11.

11

American Institute of Aeronautics and Astronautics

$39^{\text {th }}$ AIAA Fluid Dynamics Conference

Flow control: Active, passive, and closed-loop flow control: actuators 


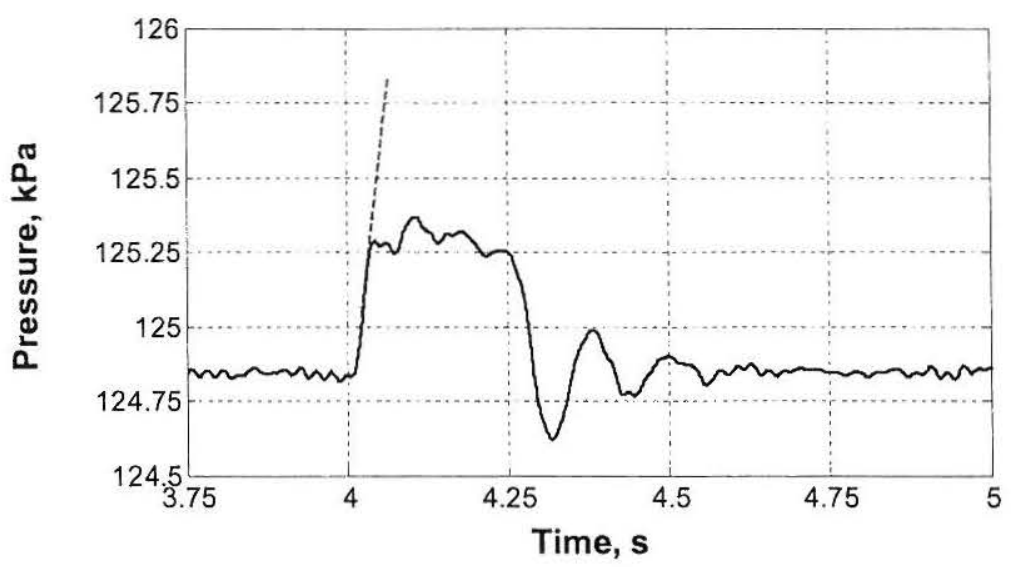

Figure 11. Pressure vs. time during a hydrostatic breakdown.

The dashed line represents the projected path of the slug had it not been overcome by hydraulic pressure. While the numerical algorithm cannot simulate the breakdown, it can predict the conditions for which it will occur. During a breakdown though, the maximum pressure is not reached.

In Perry's experiments, the failure mode was observed as a channel through the center of the slug. In the current study, the solenoids were submerged in liquid nitrogen which boiled violently as power was switched on and, therefore, the failure mode could not be seen. Nonetheless, it can be assumed that the failure mode is controlled by the magnetic field gradient, contact angle, and surface tension of the fluid. Perry's experiments used two magnetic poles on either side of the tube and tested a hydrocarbon and water as the base fluids. In the current study, the field produced by the solenoid is rotated from Perry's and generated a gradient that is highest at the edges of the solenoid instead of the middle. The contact angle of LOX on the quartz tube could not be determined, but measurements by Bembenek ${ }^{13}$ and Takeda ${ }^{14}$ showed that the surface tension of LOX in a magnetic field is lower than the fluids Perry used. Although the point at which the fluid broke down was predictable by theoretical estimation, it is not unreasonable to assume that the failure mode was different.

\section{Conclusion}

Experimental testing of the magnetohydrodynamics of a LOX slug in a closed system has been achieved. A numerical model was developed to qualitatively simulate the dynamics when the slug is displaced by pulsed magnetic fields; however, further research is needed to more accurately match the model to experimental data. The magnetic fields are induced by sequencing current through a series of solenoids that control the slug by generating pressure pulses for compression and decompression. For the test apparatus presented, the pressure pulses were on the order of $2 \mathrm{kPa}$, comparable to other researchers using ferrofluids. The simulation has proven effective in gaining understanding of the pressure differential generated for a range of slug lengths and starting positions. The study provided insight into slug motion due to a single solenoid and a series of solenoids, thus paving the way for more complex research. Overall, the understanding achieved on the magnetohydrodynamic response of a LOX slug through experimentation and simulation will be useful for future researchers seeking to design subsystems with no moving parts.

\section{Acknowledgements}

This work is supported by the Space Dynamics Laboratory and the Air Force Office of Scientific Research. Dr. Clair Batty is credited for project synthesis of the study and Dr. Steven Hansen, Dr. Byard Wood, Dr. Doran Baker, and Ms. Gayle Bowen are thanked for arranging financial provisions through the Rocky Mountain NASA Space Grant Fellowship and Tomorrow Fellowship.

\section{References}

'Rosensweig, R. E., Ferrohydrodynamics, Dover, New York, 1985, pp. 132-146.

${ }^{2}$ Odenbach, S., Ferrofluids, Springer-Verlag, Berlin, 2002, pp. 185-230. 
${ }^{3}$ Seo, K. and Park, G. S., "A Research on the Pumping Forces in the Magnetic Fluid Linear Pump," IEEE Transactions on Magnetics, Vol. 41, No. 5, 2005, pp. 1580-1583.

${ }^{4}$ Krauss, R. and Liu, M., "Pumping Fluid by Magnetic Surface Stress," New J. of Physics, Vol. 8 No. 18, 2006, pp. 1-1 I.

5Liao, W. and Chen, X., "Tunable Optical Fiber Filters with Magnetic Fluids," Applied Physics Letters, Vol. 87,2005 pp. 13.

${ }^{6}$ Hatch, A. and Kamholz, A. E., "A Ferrofluidic Magnetic Micropump," J. of Microelectromech. Syst., Vol. 10, 2001, pp. 215-221.

${ }^{7}$ Youngquist, R. C. and Immer, C. D., "Dynamics of a Finite Liquid Oxygen Column in a Pulsed Magnetic Field," IEEE Trans. on Magnetic, Vol. 39, 2003, pp. 2068-2073.

${ }^{8}$ Inman, D. J., Engineering Vibrations, $2^{\text {nd }}$ ed., Prentice Hall, New Jersey, 2001, pp. 2-88.

${ }^{9}$ White, F. M., Viscous Fluid Flow, $2^{\text {nd }}$ ed., McGraw-Hill, New York, 1991, pp. 422-424.

${ }^{10}$ Hilton, D. K. and Van Sciver, S. W., "Absolute Dynamic Viscosity Measurements of Subcooled Liquid Oxygen from 0.15 MPa to 1.0 MPa," Cryogenics, Vol. 48, 2008, pp. 56-60.

${ }^{11}$ Perry, M. P. and Jones, T. B., "Hydrostatic Loading of Magnetic Liquid Seals," IEEE Trans. on Magnetics, Vol. MAG-12, No. 6, 1976, pp. 798-800.

${ }^{12}$ Schlicting, H. Boundary-Layer Theory, $7^{\text {th }}$ ed., McGraw-Hill, New York, 1979, pp. 436-438.

${ }^{13}$ Bembenek, S. D., "Calculations of the Surface Tension of Oxygen using Molecular-Dynamics Simulations," J. of Chemical Physics, Vol. 124, No. 014709, 2006, pp. 1-4.

${ }^{14}$ Takeda, M. and Nishigaki, K., "Measurements of the Surface Tension of Liquid Oxygen in High Magnetic Fields," $J$. of the Physical Society of Japan, Vol. 61, No. 10, Oct. 1992, pp. 3631-3635. 


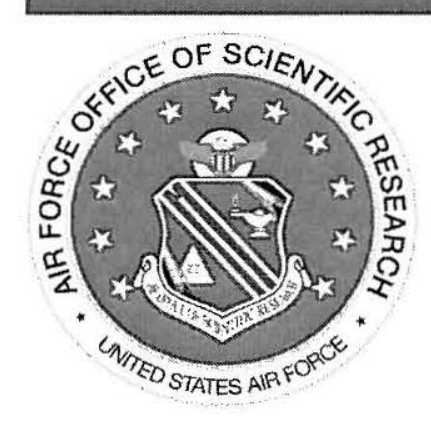

$3^{\text {th }}$ AIAA Fluid Dynamics Conference

San Antonio, TX

June 24, 2009

\title{
Characterizing the Performance of Liquid Oxygen in a Magnetic Fluid Management System
}

\author{
Jeffrey C. Boulware \\ Heng Ban \\ Utah State University \\ Scott Jensen \\ Steve Wassom \\ Space Dynamics Laboratory
}

UtahStateUniversity 


\section{Magnetic Fluids}

- Most research performed on ferrofluids

- Colloidal suspensions of ferromagnetic nanoparticles

- Oxygen is a paramagnetic fluid

- Attracted to a magnetic field, but does not create its own

- Susceptibility is inversely proportional to temperature

- Curie's Law

- Youngquist performed only known MHD tests on LOX

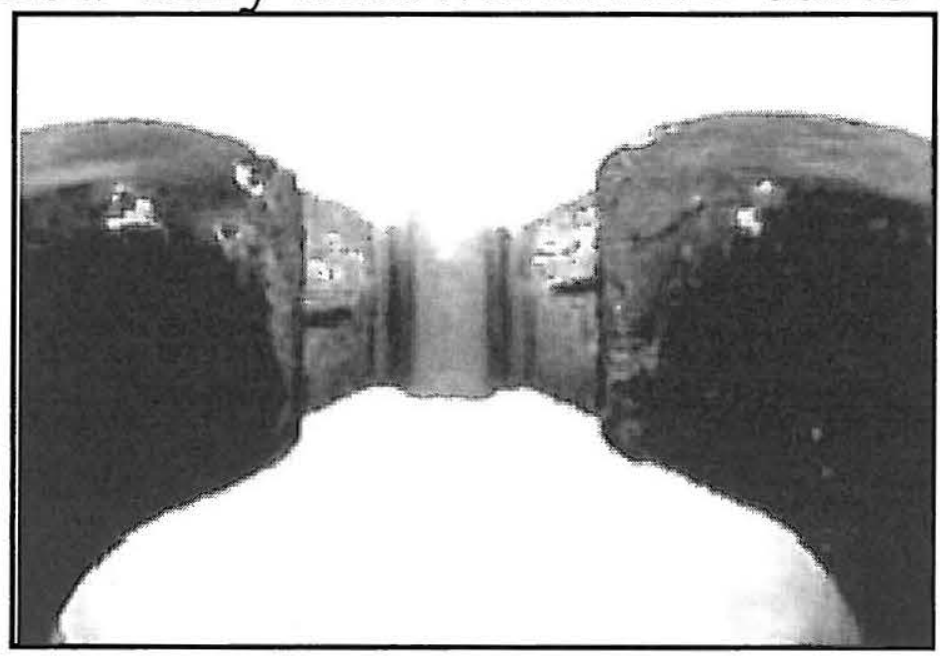




\section{Experimental Principle}

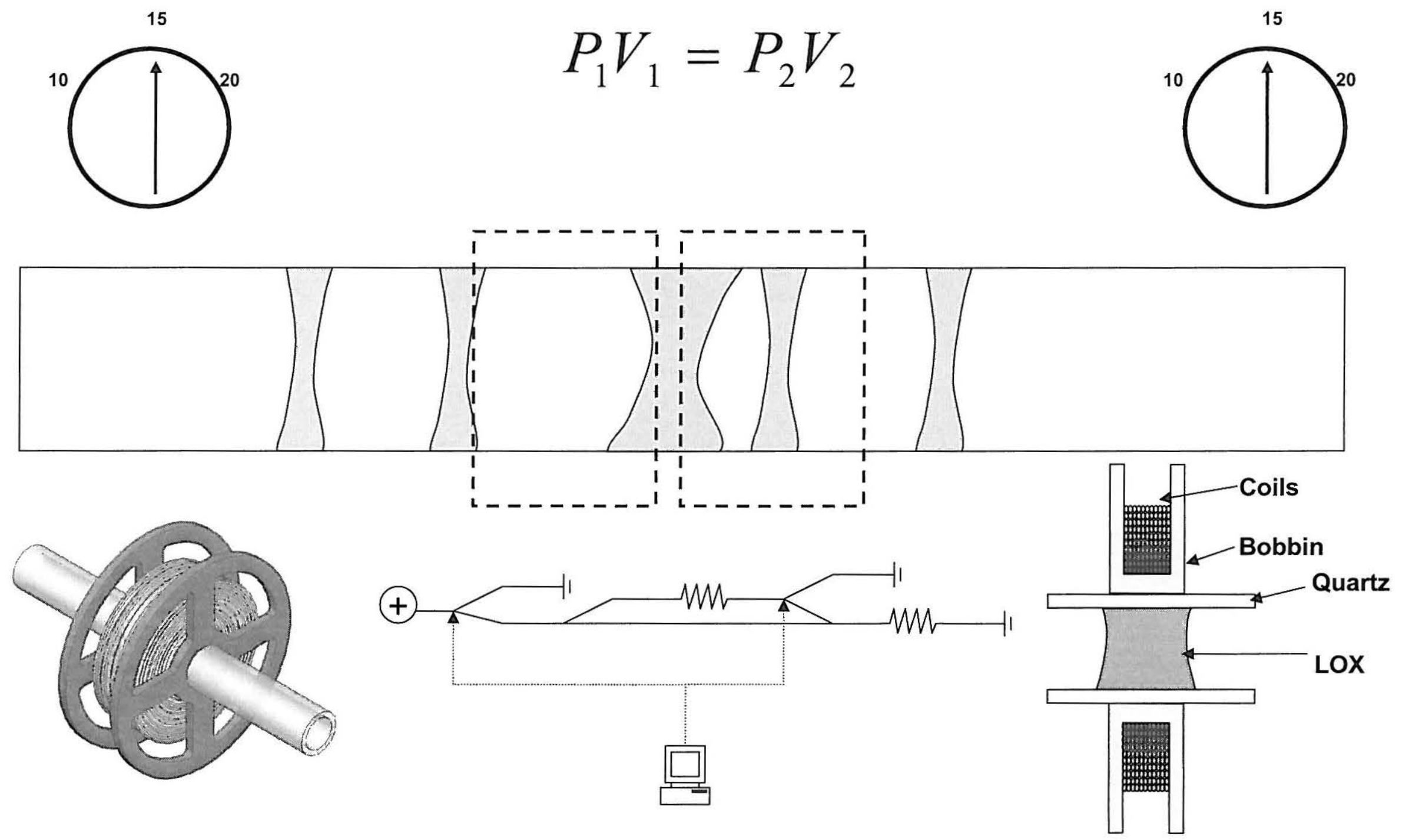




\section{Experiment Setup}

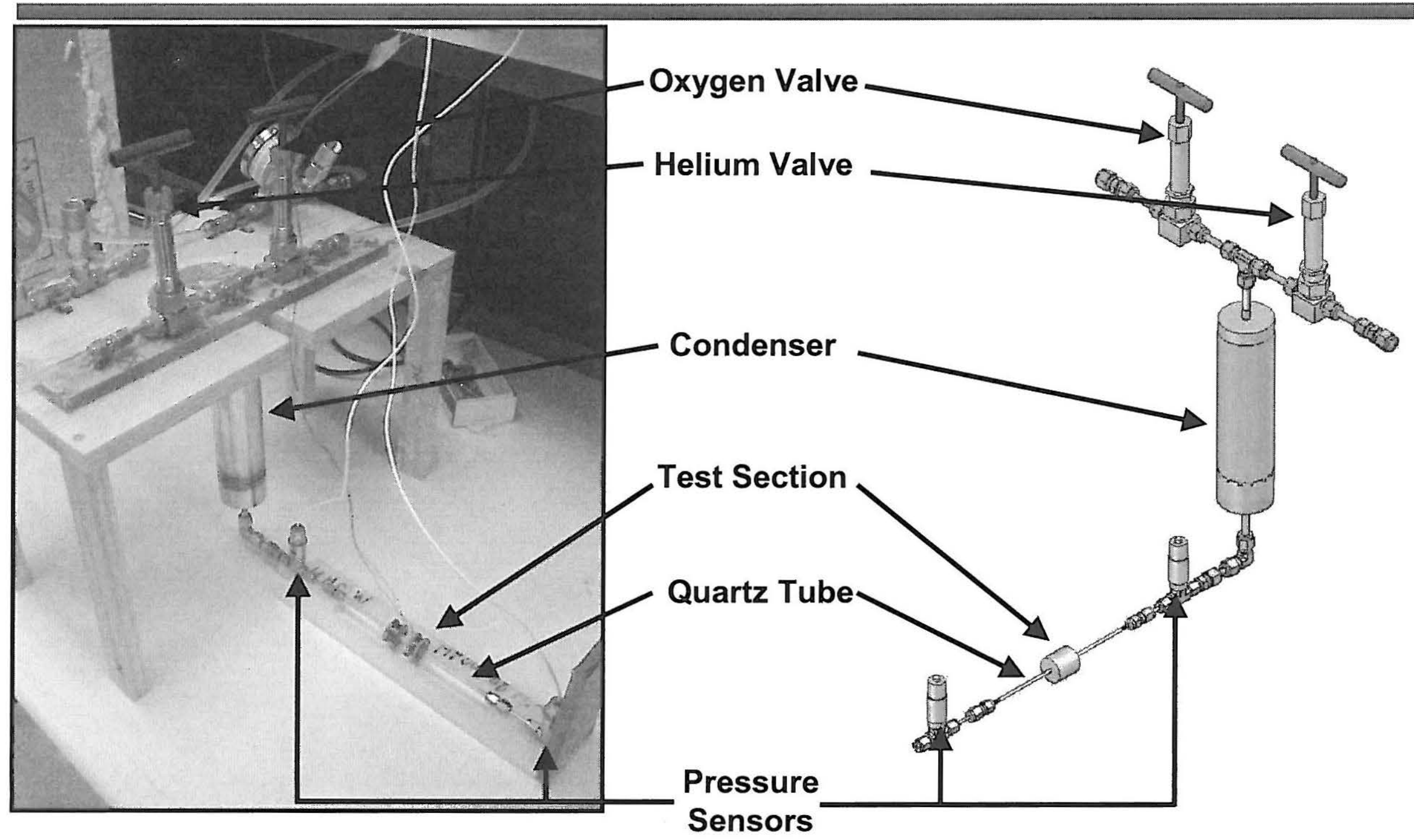




\section{Theory - Force Balance}

$$
\begin{aligned}
& m \ddot{x}=F_{P}(x)+F_{M}(x)+F_{S}(\dot{x}) \\
& F_{P}=\pi a^{2} \Delta p \\
& F_{M}=\frac{\pi a^{2}}{2 \mu_{0}} \frac{\chi}{(1+\chi)^{2}}\left(B_{x, U S}^{2}-B_{x, D S}{ }^{2}\right) \\
& F_{S}=2 \pi a L \tau_{W}
\end{aligned}
$$
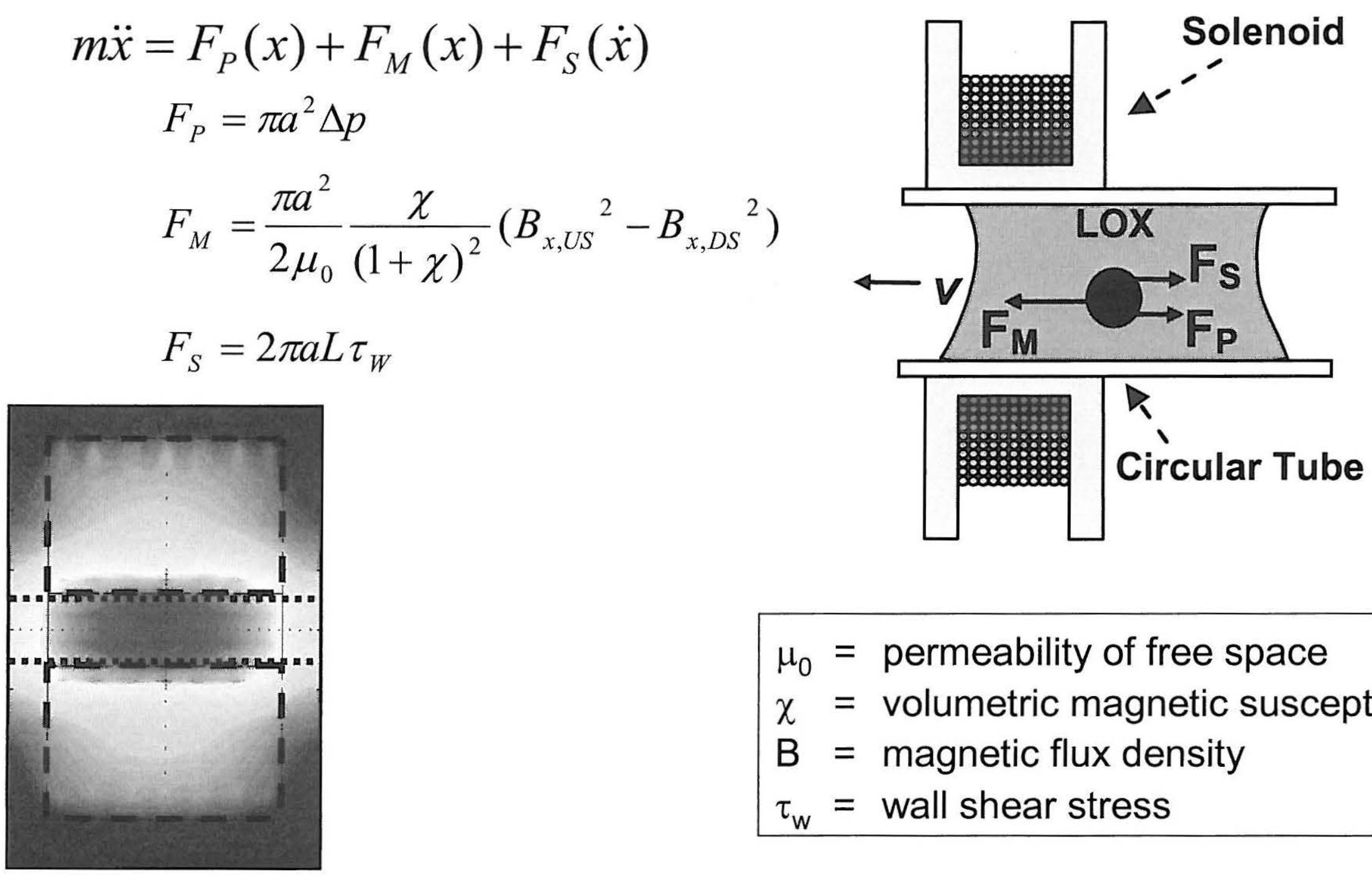

$\mu_{0}=$ permeability of free space

$\chi=$ volumetric magnetic susceptibility

$\mathrm{B}=$ magnetic flux density

$\tau_{\mathrm{w}}=$ wall shear stress 


\section{Numerical Model}

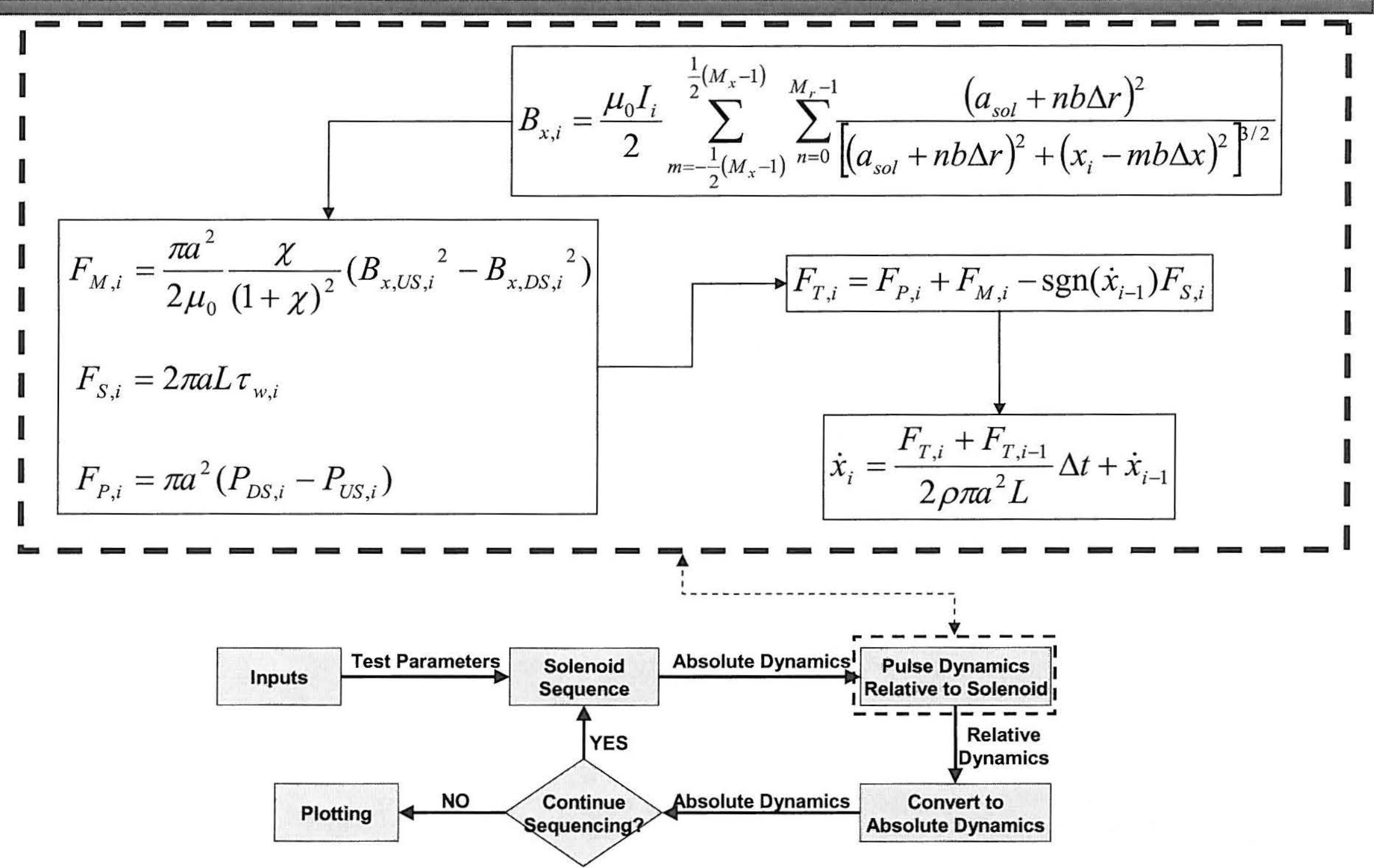




\section{Results - Single Pulse}

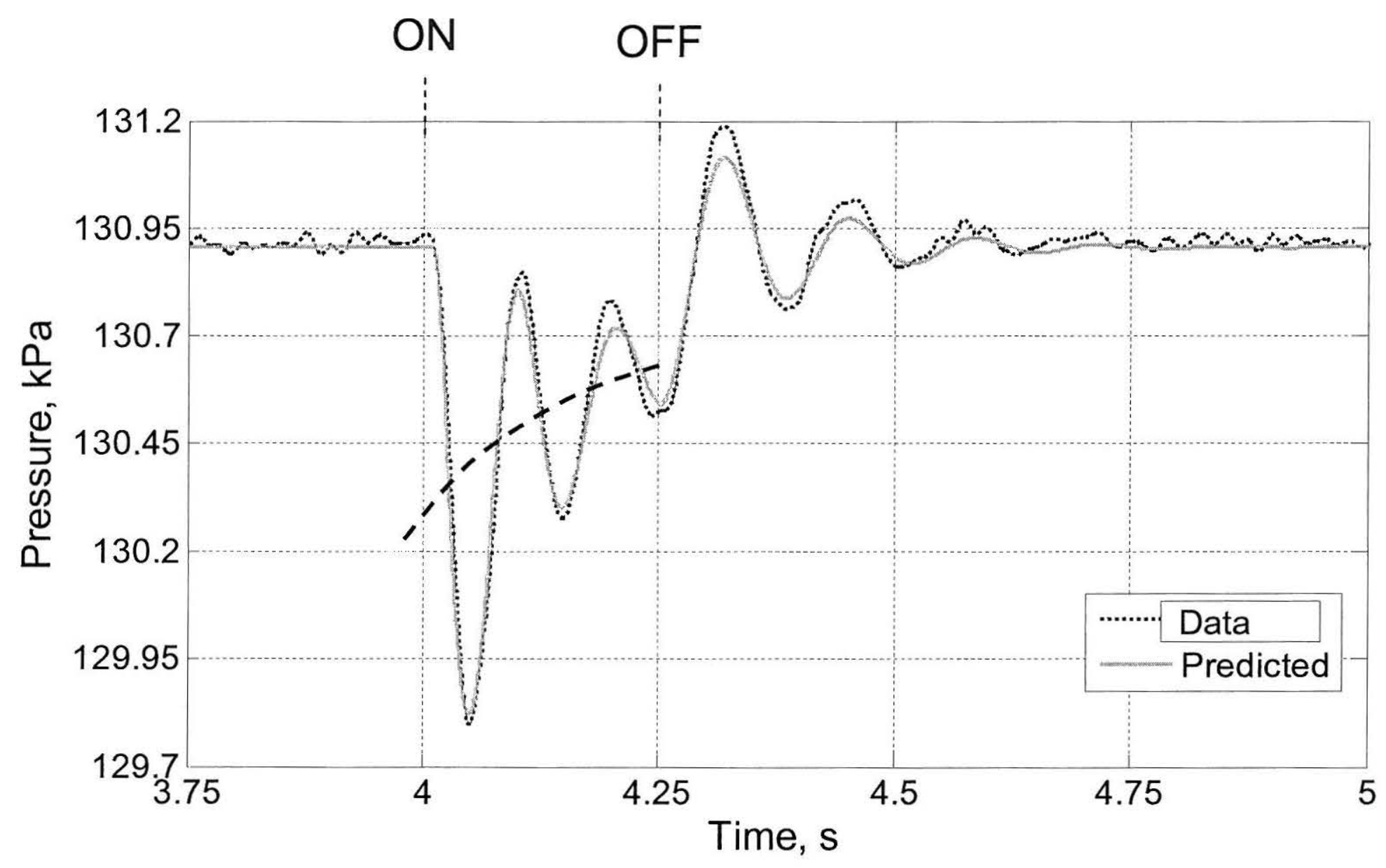




\section{Results - Single Pulse}

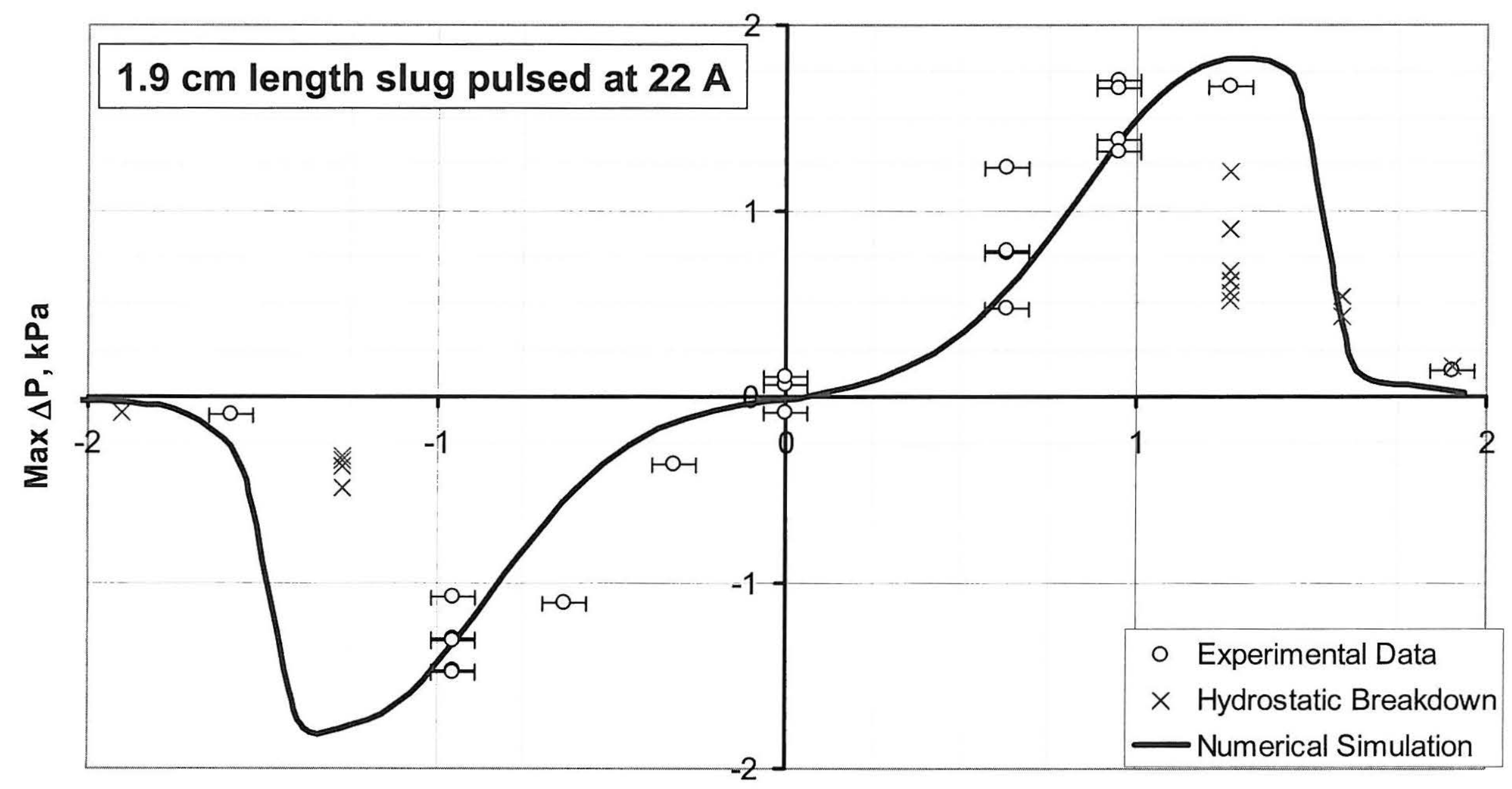

Slug Center Position, cm 


\section{Results - Single Pulse}
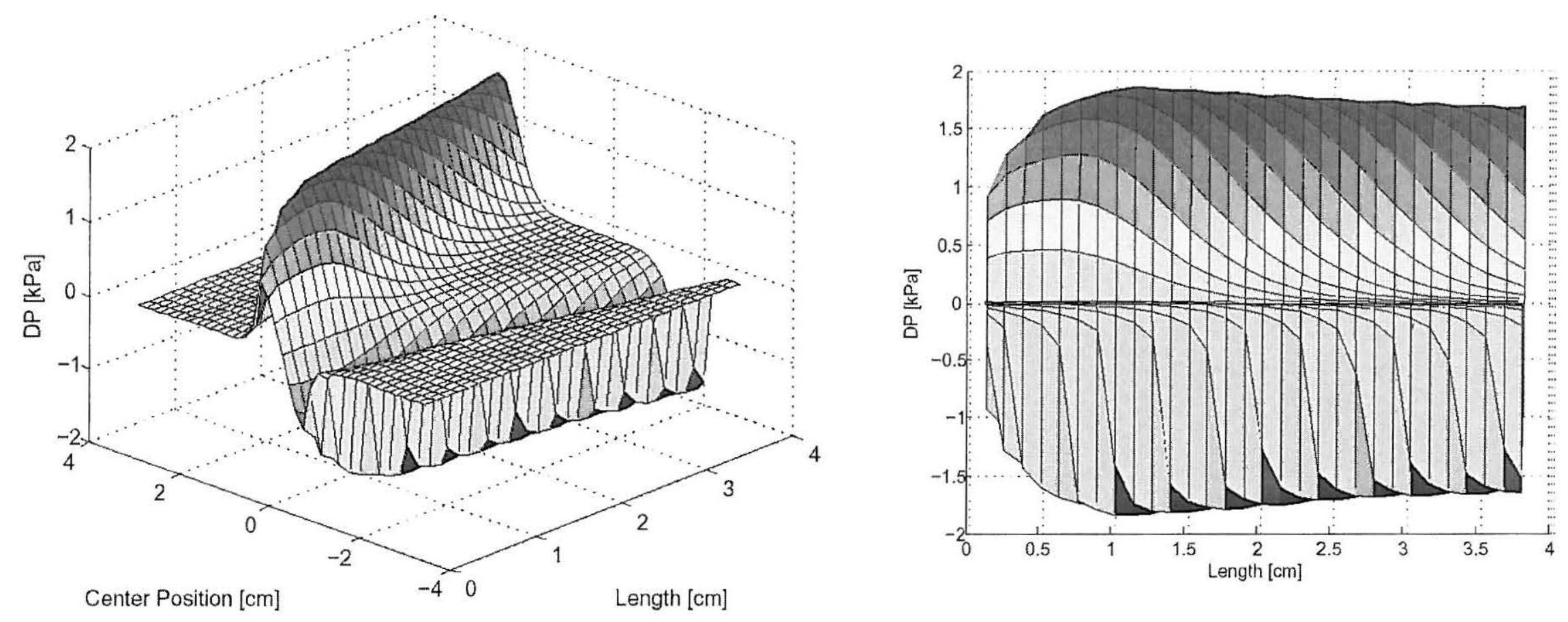


\section{Results - Multiple Pulses}

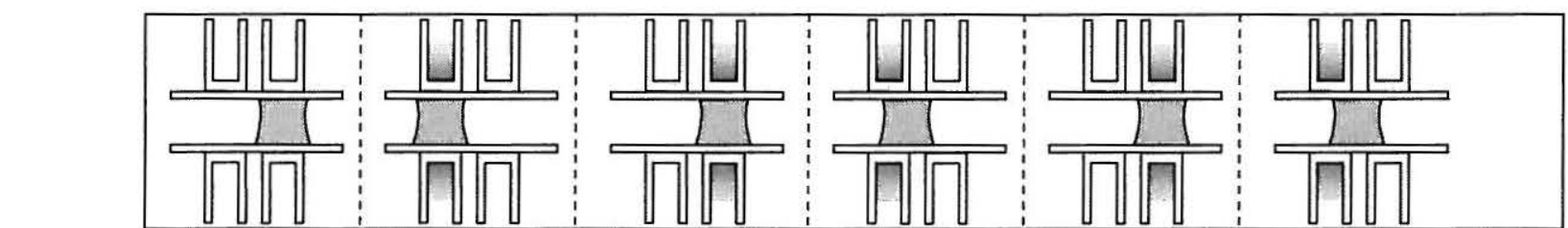

Measured Downstream Pressure, $\mathrm{kPa}$

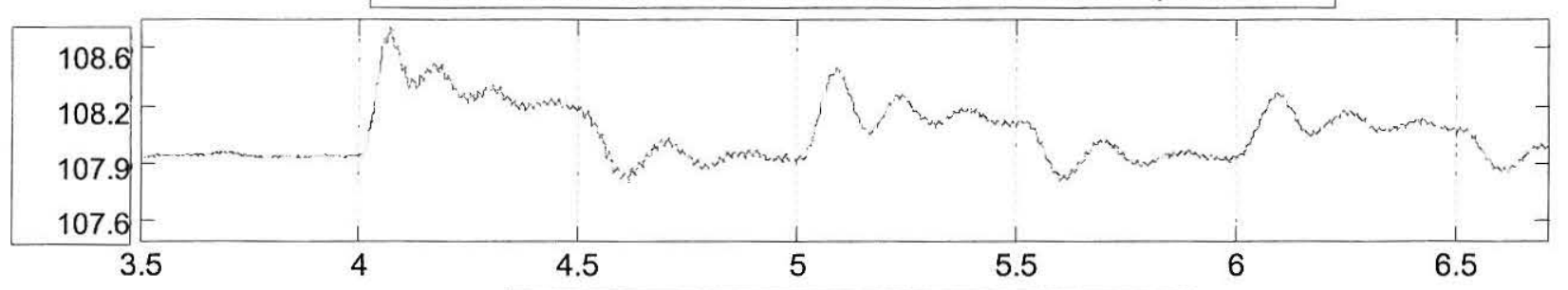

Estimated Displacement, $\mathrm{cm}$

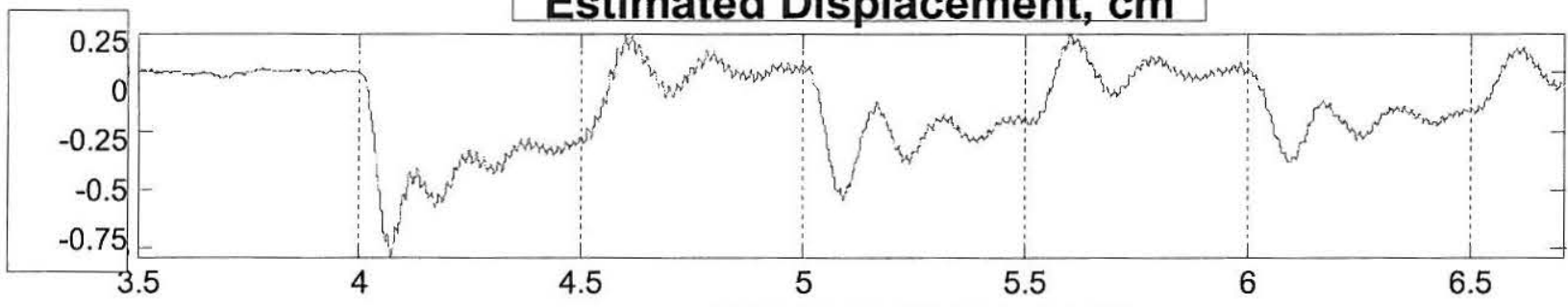

Applied Voltage, V

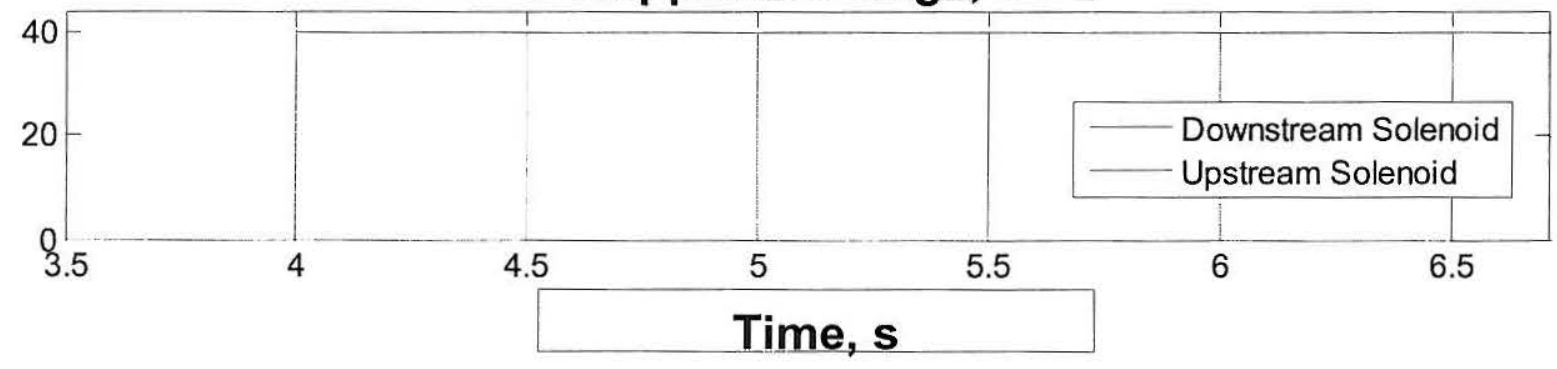




\section{Results - Hydrostatic Breakdown}

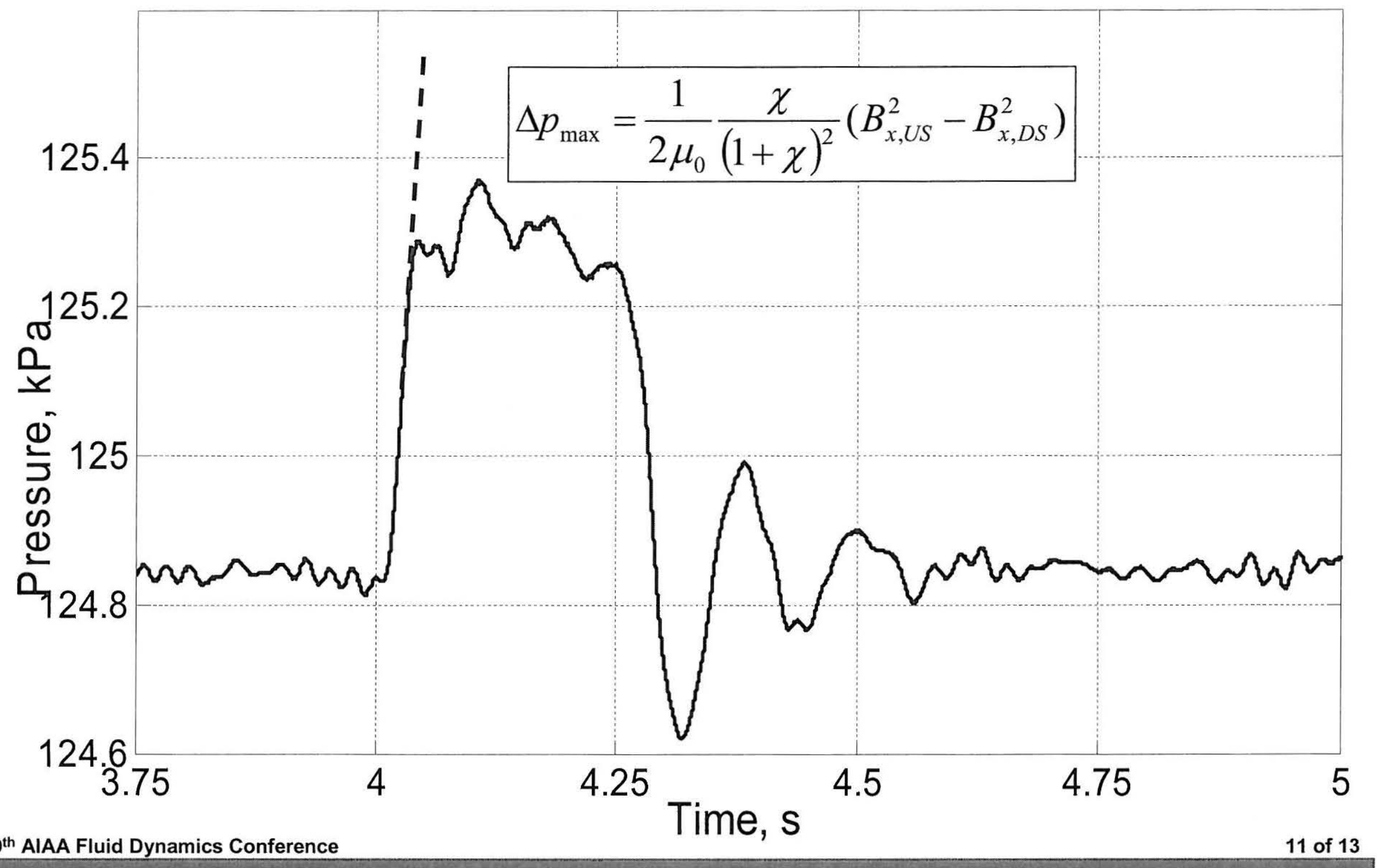




\section{Conclusions}

- LOX was controllably displaced using magnetic fields

- Its single and multiple pulse dynamics were successfully simulated

- The hydrostatic breakdown was observed through the data at a predictable point. 


\section{Acknowledgements}

- Space Dynamics Laboratory

- Air Force Office of Scientific Research

- Dr. Clair Batty

- Dr. Steven Hansen

- Dr. Byard Wood

- Dr. Doran Baker

- Ms. Gayle Bowen

- Rocky Mountain NASA Space Grant Fellowship

- Tomorrow Fellowship 